\title{
The Effect of the Gas Flow-Rate on the Radial Structure of a Torch-Like Helium Plasma
}

\author{
R. Álvarez, M. C. Quintero, and A. Rodero
}

\begin{abstract}
The effect of the gas flow-rate on the radial structure of a torch-like helium microwave plasma has been studied by measuring the radial distributions of emission intensity of spectral lines from helium and from the air that surrounds the discharge. It has been found that increasing the gas flow-rate leads to an increase of the maximum of the spectral lines and a shift of these emission peaks toward the plasma edge. Apart from that, it is found that the entrainment of air into the plasma increases with the flow-rate.
\end{abstract}

Index Terms-Abel inversion, helium, plasma measurements, plasma properties, plasma torches, spectroscopy.

M ICROWAVE induced plamas (MIPs) have an increasing number of industrial applications. In torch-like MIPs sustained at atmospheric pressure, the air surrounding the discharge enters the plasma via diffusion and turbulent mixing, which can affect the plasma properties [1].

In this paper, the axial injection torch (AIT) has been used to produce a torch-like helium MIP at atmospheric pressure. The plasma created by the AIT has a small diameter $(\sim 1 \mathrm{~mm})$ and a variable length that depends on the plasma conditions of gas flow rate and injected microwave power, ranging between 2 and $15 \mathrm{~cm}$. The effect of the gas flow-rate on the plasma radial structure has been studied by measuring the radial distribution of the emission intensity from two different emission lines: the $388.9 \mathrm{~nm}$ HeI line and the $391.4 \mathrm{~nm} \mathrm{~N}_{2}^{+}$line. The HeI line provides information about the helium radial distribution, while the $\mathrm{N}_{2}^{+}$line (the bandhead of the rotational band corresponding to the first negative system ( $0-0)$ of the nitrogen molecular ion) provides information about the radial distribution of the molecular species present in the plasma due to its entrainment from the surrounding air.

These lines have been measured at $1 \mathrm{~mm}$ above the AIT nozzle tip, for $600 \mathrm{~W}$ of supplied microwave $(2.45 \mathrm{GHz})$ power and different conditions of helium flow-rate: from $0.5 \mathrm{~L} / \mathrm{min}$ to $2.5 \mathrm{~L} / \mathrm{min}$.

In order to obtain the radial distributions of the line intensities, an Abel inversion has to be applied on the experimental data. The Abel inversion [2] allows to obtain the radial distribution of emission intensity, $\varepsilon(\mathrm{r})$, from the lateral distribution of the emission intensity of an axially symmetric source integrated along the line-of-sight, $I(x)$

$$
\varepsilon(r)=-\frac{1}{\pi} \int_{r}^{R} \frac{I^{\prime}(x)}{\sqrt{x^{2}-r^{2}}} d x
$$

Manuscript received July 2, 2004; revised November 9, 2004. This work was supported by the Spanish Ministry of Science and Technology under Project PPQ 2001-2537.

The authors are with the Department of Physics, University of Córdoba, 14071 Córdoba, Spain.

Digital Object Identifier 10.1109/TPS.2005.845324

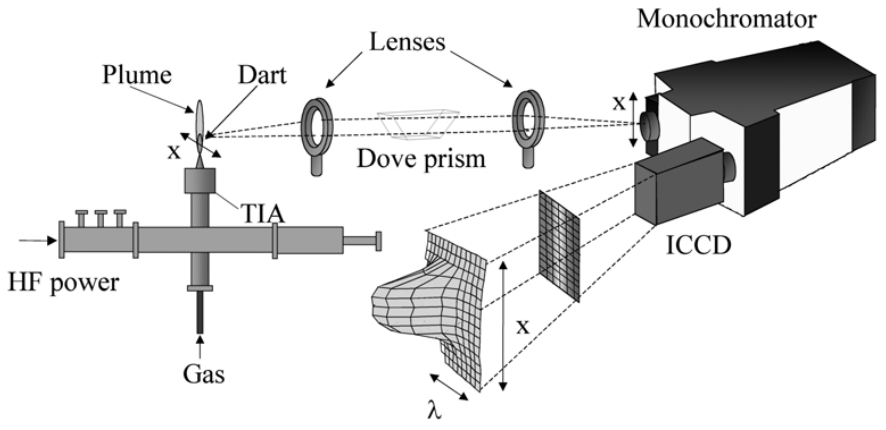

Fig. 1. Experimental setup.

where $R$ is the plasma radius and $I^{\prime}$ is the derivative of $I(x)$ with respect to $x$, the lateral position.

The experimental setup (Fig. 1) is designed to measure the lateral distribution $I(x)$ at any desired wavelength: a 1:3 magnified image of the plasma is rotated $90^{\circ}$ and focused on the $60-\mu \mathrm{m}$ vertical entrance slit of a monochromator. This allows the selection of a line-of-sight integrated plasma slice to enter the monochromator. At the exit of the monochromator, an intensified CCD camera collects the wavelength-dispersed image with its two-dimensional (2-D) array of photosensitive pixels. The position of the plasma edge $(r=R)$ was considered to be where the helium emissivity was below $1 \%$ of its maximum. The measurements show that the lateral positions of maximum emission intensity from the $\mathrm{HeI}$ and the $\mathrm{N}_{2}^{+}$lines point to a difference in the shape and maxima of the radial emission distributions. This is confirmed when the Abel inversion is performed: the helium presents an off-center maximum, with low emission values at the plasma axis, while the $\mathrm{N}_{2}^{+}$coming from the air shows a narrow emission peak at the edge of the plasma and negligible emission values at the rest of radial positions. The $\mathrm{N}_{2}^{+}$emission drops at the radial positions where the HeI emission starts to rise, which probably indicates that at those positions the plasma has energy enough to dissociate the $\mathrm{N}_{2}^{+}$molecule. Fig. 2 shows the spatial distributions of the $\mathrm{HeI}$ and $\mathrm{N}_{2}^{+}$bandhead emission lines for different gas flow-rate conditions: the inner maximum corresponds to the $\mathrm{HeI}$ line, while the outer maximum corresponds to the $\mathrm{N}_{2}^{+}$line. A first conclusion to be drawn from this figure is that the shape of the plasma, as described by the HeI line spatial distribution, clearly changes with the gas flow-rate, being the radial maximum displaced toward the plasma edge with the increase in the gas flow-rate. It can also be seen that the emission intensity of $\mathrm{N}_{2}^{+}$increases with the flow-rate, which is probably caused by the increase in the turbulent mixing of the air with the plasma that is produced when the gas flow-rate is increased. Also, the plasma radius increases lightly with the gas flow-rate. 


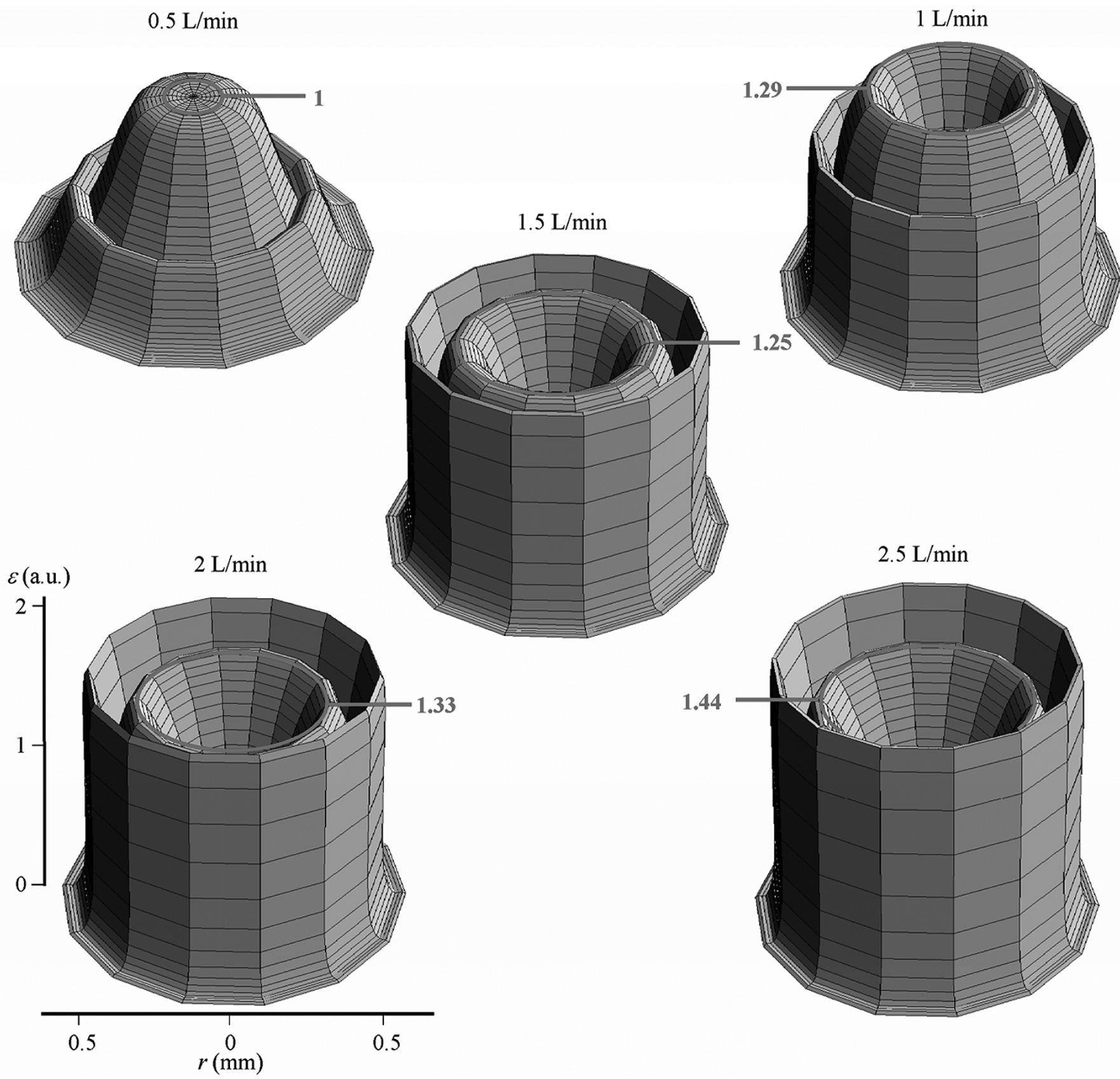

Fig. 2. Variation of the spatial distribution of the emission intensity $\varepsilon$ of a HeI line (inner maximum) and a $\mathrm{N}_{2}^{+}$line (outer maximum) with the He flow-rate. Maximum of the HeI distribution and its value (in a.u.) have been marked in the figure.

\section{REFERENCES}

[1] J. Jonkers, A. Hartgers, L. J. M. Selen, J. A. M. van der Mullen, and D. C. Schram, "The influence of nitrogen entrainment on argon plasmas created by the 'Torche a Injection Axiale' (TIA)," Plasma Sources Sci. Technol., vol. 8, pp. 49-57, 1999.
[2] R. Alvarez, A. Rodero, and M. C. Quintero, "An Abel inversion method for radially resolved measurements in the axial injection torch," Spectrochim. Acta Part B, vol. 57, pp. 1665-1680, 2002. 